\title{
DOCTRINA
}

\section{Zero-rating y la neutralidad de la red en Chile}

\author{
Zero-rating and net neutrality in Chile
}

\author{
Marco Correa Pérez \\ Wikimedia Chile
}

RESUMEN La regulación chilena de neutralidad de la red ha sido ampliamente analizada a nivel mundial, ya que constituye el primer ejemplo de ley que consagra este principio, que tiene como objeto evitar la discriminación arbitraria al tráfico de internet. Uno de los principales desafíos a esta ley ha sido el zero-rating, en que los proveedores de telecomunicaciones priorizan ciertas aplicaciones mediante la gratuidad de los datos consumidos en ellas. A pesar de que estas prácticas fueron calificadas como una trasgresión de la neutralidad de la red por el ente regulador, la Subsecretaría de Telecomunicaciones (Subtel), éstas siguen siendo ofrecidas en los planes de telefonía móvil. Este artículo analiza el estatus jurídico del zero-rating y la evolución del criterio de la Subtel.

PALABRAS CLAVE Neutralidad en la red, zero-rating, telefonía móvil, telecomunicaciones.

ABSTRACT The Chilean net neutrality regulation has been widely analyzed worldwide, as it is the first law in the world to recognize this principle, which aims to prevent arbitrary discrimination of internet traffic. One of the main challenges in interpreting and implementing this law within Chile has been the regular practice of zero-rating, in which certain telecommunications providers prioritize certain applications through free data. Although this practice was qualified as a breach of net neutrality by the regulatory body, the Subsecretariat of Telecommunications (Subtel), zero-rating is still practiced by mobile telephone companies as part of their subscription offers. This article seeks to analyze both the legal status of zero-rating in Chile and the evolution of the Subtel criteria.

KEYWORDS Net neutrality, zero-rating, mobile telephony, telecommunications. 


\section{Introducción}

El abrupto cambio en el criterio del ente regulatorio de las telecomunicaciones de los Estados Unidos (la Federal Communications Commission) sobre la neutralidad de la red, que se materializó en la decisión de revertir la normativa federal de este principio a fines de 2017, reavivó el debate sobre la regulación de la gestión del tráfico de internet no solo en ese país, sino que en todo el mundo. Chile no ha estado exento de la discusión, ${ }^{1}$ toda vez que es el país que se jacta de tener la primera legislación que consagró la neutralidad de la red, la Ley 20.453 de 2010.

Por ello resulta oportuno revisar la aplicación de la neutralidad de la red en Chile, con un especial enfoque en la práctica comercial denominada zero-rating o tasa cero, cuya conformidad con dicho principio ha sido intensamente debatida en derecho comparado.

El zero-rating se ha consolidado en la última década como una de las ofertas más populares hechas por las empresas de telecomunicaciones alrededor del mundo. Desde luego que Chile no ha estado exento de esa tendencia mundial, ya que el zerorating ha sido habitualmente publicitado por compañías de telefonía móvil bajo el concepto de «redes sociales gratis», debido a que se concentra en plataformas que involucran el intercambio de contenidos entre usuarios finales, como Facebook o Whatsapp.

El fenómeno del zero-rating cobra especial relevancia en Chile porque comenzó a ser ofrecido después de dos años de vigencia de la normativa de neutralidad de la red, y a pesar de que la Ley 20.453 establece que los prestadores de servicios de internet (conocidos como ISP, por internet service providers) no deben discriminar entre diferentes aplicaciones, servicios y contenidos a los que acceden sus usuarios. Esta obligación a lo menos permite cuestionar la legalidad de la tasa cero, toda vez que dicha práctica puede caracterizarse como una forma de discriminación entre aplicaciones. De hecho, ese fue el criterio que adoptó en 2014 el regulador chileno, la Subsecretaría de Telecomunicaciones (Subtel), cuando ofició a las empresas telefónicas para que adecuaran sus ofertas de «redes sociales gratis» al marco normativo vigente.

A pesar de ello, las compañías telefónicas no solo continuaron ofreciendo promociones de zero-rating, sino que han ido en aumento. Por esto, llama la atención que la literatura chilena carezca de estudios respecto de la situación jurídica del zero-rating en el país, ya que los que existen provienen en su mayoría de publicaciones y autores

\footnotetext{
1. La decisión del regulador estadounidense motivó a que el regulador chileno de telecomunicaciones convocara a los diversos actores interesados (industria de las telecomunicaciones, proveedores de contenidos, sociedad civil y academia) a conformar una mesa de trabajo sobre neutralidad de la red en diciembre de 2017.
} 
extranjeros que analizan las regulaciones de varios países, ${ }^{2}$ en los que se tiende a obviar antecedentes de suma importancia que derivan en la conclusión, errónea a nuestro juicio, de que en Chile se prohíbe de manera absoluta la tasa cero (véase Marsden, 2016: 14-15; Gharakheili, Vishwanath y Sivaraman, 2016; Rossini y Moore, 2015: 3; Layton y Elaluf-Calderwood, 2015: 13). Esta es una de las principales motivaciones para la redacción del presente estudio.

En primer lugar, haremos un breve repaso de los conceptos de neutralidad en la red y zero-rating, la discusión sobre la compatibilidad de ambos conceptos y las posibles respuestas a este eventual conflicto. En segundo lugar, nos centraremos en el caso chileno, al analizar la Ley 20.453 para determinar si contiene una prohibición (expresa o tácita) a la tasa cero, y el criterio que ha tenido la Subtel respecto de dicha práctica comercial desde la promulgación, que emana tanto del reglamento como de otras fuentes.

\section{La neutralidad en la red}

El concepto de neutralidad en la red fue acuñado por el profesor Tim Wu (2003), quien recogió lo que había sido hasta ese momento la discusión sobre qué implicaba una internet abierta. Desde entonces el tema no solo ha sido estudiado y analizado ampliamente por la academia, sino que se ha vuelto uno de los de mayor relevancia en la discusión de las políticas públicas sobre acceso a internet y regulación de la industria de las telecomunicaciones.

A pesar de la amplia literatura sobre la materia, la definición de este principio sigue siendo controvertida, ya que existen distintas visiones sobre sus objetivos y la forma en que éstos se obtienen, particularmente en relación con la restricción de las facultades de los ISP para gestionar el tráfico de internet de los usuarios finales (Barata, 2012).

Nos permitimos citar algunas de las definiciones que se han dado a la neutralidad de la red. Para Wu (2003: 142), el objetivo de la neutralidad de la red «es otorgar a los usuarios el derecho de utilizar archivos adjuntos o aplicaciones de red no dañinos y brindar a los innovadores la libertad correspondiente para suministrarlos». Marsden (2010: 29) definió neutralidad en la red como «un principio relativo al acceso del usuario final de internet a los proveedores de contenido, y a la potencial discriminación en ese acceso, donde el ISP del usuario final u otro ISP bloquea ese acceso parcial o totalmente, incluso sujeto a tarifas especiales». Según Jordan y Ghosh (2010: 2), «la neutralidad de la red representa la idea de que los usuarios de internet tienen derecho a un servicio que no discrimine en función del origen, el destino o la pro-

2. Una de las pocas excepciones es un informe dirigido por la ONG Derechos Digitales, en el que fue analizado el zero-rating en Chile, Colombia, Brasil y México (Castro, Pereira da Silva y Viollier, 2017). 
piedad del tráfico de internet». De acuerdo con los lineamientos del Organismo de Reguladores Europeos en Comunicaciones Electrónicas, la neutralidad de la red busca «salvaguardar el trato igualitario y no discriminatorio del tráfico en la provisión de servicios de acceso a internet y los derechos de los usuarios finales relacionados». ${ }^{3}$

A partir de estas descripciones, podemos seguir que el principal objetivo de la neutralidad de la red es, en palabras simples, impedir el trato discriminatorio al tráfico de internet. La regulación de dicho tráfico, con el fin de garantizar la neutralidad, involucra los intereses de tres clases de sujetos. En primer lugar, los ISP, quienes están obligados a proveer el servicio de internet de forma igualitaria y no discriminatoria, independientemente al origen, propiedad o destino del tráfico. En segundo lugar, los usuarios finales de internet, a quienes la neutralidad garantiza un acceso y ejercicio libre de sus actividades en línea. Y, en tercer lugar, los proveedores de los contenidos en línea («innovadores», en la definición de Tim Wu), quienes se verán beneficiados por la no discriminación del tráfico para llegar en igualdad de condiciones a los usuarios finales.

El ámbito de aplicación de la neutralidad de la red ha ido evolucionando con el paso del tiempo. En un comienzo se configuró como una respuesta a la conducta de los ISP de ejecutar deliberadamente bloqueos, interrupciones e interferencias a los contenidos y servicios, conductas que en la actualidad son ampliamente aceptadas como trasgresiones a aquel principio. Éstas son las que busca evitar la llamada neutralidad «negativa» (Marsden, 2016: 7).

Sin embargo, pronto la neutralidad de la red se extendió a otras actuaciones de los ISP, que han intentado replicar modelos de negocios que en otras industrias, como la televisión paga, son indiscutidamente permitidas. Un ejemplo de estos modelos importados al internet son los llamados «jardines vallados» (walled gardens), en los que el proveedor del servicio puede establecer un trato diferencial de los contenidos a los que acceden los consumidores (Marsden, 2016: 7). Un ejemplo de jardín vallado es la decisión de los proveedores de televisión satelital sobre qué canales puede ver el cliente y a qué precio, para lo cual establece categorías de planes con distintos contenidos. En el entorno de internet, estas prácticas de selección o priorización de contenidos son lo que busca evitar la llamada neutralidad «positiva».

Las prácticas que prohíbe la neutralidad positiva son las más controvertidas en cuanto a su regulación, pues parte de la doctrina niega su incompatibilidad con la neutralidad de la red. Una de ellas es el zero-rating, la cual analizaremos en la próxima sección.

3. «BEREC guidelines on the implementation by national regulators of European net neutrality rules», Body of European Regulators for Electronic Communications, 30 de agosto de 2016, p. 4, disponible en https://bit.ly/2bOVMIZ. 


\section{El zero-rating}

\section{Concepto y modelos}

El zero-rating o tasa cero 4 consiste en la oferta por los proveedores de servicios de internet de exención de la cobranza de los costos a los usuarios finales por la utilización de datos de internet relativos a ciertos servicios o contenidos específicos.

Así, por ejemplo, se denomina tasa cero a las ofertas en que los datos utilizados en los sitios web o aplicaciones priorizados no son cobrados a los consumidores de internet de prepago, o no son descontados de la cuota mensual a los clientes de pospago con servicios de datos limitados. En ambos casos, se trata de servicios cuyo costo está ligado directamente a los datos usados durante un periodo determinado, también conocidos como data cap, que se efectúan principalmente en las conexiones móviles. En los data caps, el consumo de datos tiene un costo de oportunidad, que está determinado por el valor que tiene el acceso al contenido cuando se agota el límite total de los datos (Comisión Europea, 2017: 1).

El zero-rating no tiene aplicación práctica en el caso de los servicios de datos ilimitados, ${ }^{5}$ en el cual los usuarios pueden descargar todo lo que necesiten, con la única restricción de la velocidad de la conexión (Marsden, 2016: 7). Sin embargo, cabe tener en consideración que el alto costo de los planes móviles ilimitados —en comparación con los data caps - en muchos de los países con una alta o mediana penetración de internet, o el precio prohibitivo del acceso a internet en general, cualquiera sea su tipo, en los países con bajo acceso a la red, hacen que las ofertas zero-rating resulten atractivas para una gran parte de los usuarios de internet del mundo. ${ }^{6}$ De ahí que incluso haya programas de tasa cero que benefician a personas que no tienen ningún plan de internet, como Free Basics de Facebook (KeenanAlspector, 2016).

La complejidad del debate sobre el zero-rating está también condicionada por la

4. Estas son las denominaciones más comunes para esta práctica. Según Gharakheili, Vishwanath y Sivaraman (2016), otros términos utilizados son: sponsored data («datos patrocinados»), unmetered content («contenido sin medir») y toll-free data («datos gratuitos»). Sin embargo, sponsored data no es sinónimo de zero-rating, ya que es un tipo de tasa cero.

5. Existen servicios de datos «ilimitados» en que, después de que se consume cierto volumen de datos, el tráfico de internet no se detiene, sino que solo se reduce la velocidad de conexión. Para efectos de este estudio no los consideramos ilimitados, porque funcionan de modo similar a los data caps al fijar un límite de datos para otorgar un servicio de la calidad que el cliente contrata.

6. Según la International Telecommunications Union, en los países de bajo índice de desarrollo humano, el costo de una conexión fija de banda ancha estándar (1 GB mensual) es 2,6 veces más cara que la conexión a internet móvil. Esto significa que, para una gran parte de los habitantes del mundo, el teléfono sea la única opción para acceder a la red. «ICT facts and figures 2017», International Telecommunications Union, disponible en http://bit.ly/2MdwCmn. 
amplia gama de prácticas que se ven englobadas en este concepto. Con el objeto de simplificar el análisis, presentamos dos criterios para su clasificación:

- Patrocinio: Se refiere a quién asume el costo de los datos consumidos por los usuarios finales. El patrocinador de la tasa cero puede ser tanto el proveedor de internet como el proveedor de los contenidos.

- Contenidos: Se refiere a qué contenidos son priorizados por la tasa cero. Puede aplicarse a los datos generados por un único contenido (aplicación o sitio web) o a un paquete de contenidos seleccionados. En este último caso, puede tratarse de aplicaciones de utilidad similar o diferente.

A partir de la combinación de estos factores, pueden hallarse diferentes modelos de zero-rating, en que los más típicos son de un único sitio, patrocinado, compuesto, de una categoría de servicios y «falso», sintetizados en la tabla 1.

- Único sitio: Fue el primer tipo de tasa cero y consiste en el otorgamiento de acceso sin costo a un solo servicio (sitio web o aplicación) por parte del ISP. Por regla general, el ISP es quien patrocina la tasa cero en este modelo, a pesar de que exista un acuerdo con el proveedor del contenido priorizado (KeenanAlspector, 2016: 10). Este último caso es el de Wikipedia Zero, un programa de acceso gratuito a la enciclopedia Wikipedia que fue promovido por la Fundación Wikimedia en varios países mediante alianzas con empresas telefónicas. ${ }^{7}$

- Datos patrocinados (sponsored data) o «patrocinado»: Es uno de los modelos de zero-rating más analizados por la doctrina. Se caracteriza porque el costo de los datos usados es asumido por el proveedor del contenido, que paga por dichos costos al ISP. Este modelo debutó en los Estados Unidos en 2014, cuando AT\&T ofreció a las empresas pagar por los datos que consumían sus clientes en la descarga y uso de aplicaciones de prueba o en los anuncios en video de sus contenidos (Ard, 2016: 996).

- Compuesto (compound zero-rating): Modelo en que una empresa -o un conjunto de ellas- se asocia con un proveedor de contenidos de internet para otorgar a sus clientes acceso sin costo a un paquete de sitios y aplicaciones seleccionadas. Dicho acuerdo puede o no involucrar un pago al ISP por parte del proveedor del contenido (Carrillo, 2016: 378-379). Uno de los principales ejemplos de zero-rating compuesto es el ya mencionado programa Free Basics

7. En febrero de 2018, la Fundación Wikimedia anunció que descontinuaría Wikipedia Zero, justificándose en la pérdida de interés y en los cambios en el mercado del internet móvil, sin invocar entre sus razones los cuestionamientos a su programa por activistas de la neutralidad de la red. «Building for the future of Wikimedia with a new approach to partnerships», Blog de Wikimedia Foundation, 16 de febrero de 2018, disponible en http://bit.ly/2MfB5VU. 
Tabla 1. Tipos de zero-rating

\begin{tabular}{|lll|}
\hline Tipo de zero-rating & Patrocinio & Contenido \\
\hline Único sitio & ISP & Un sitio o aplicación \\
\hline Patrocinado & Proveedor de contenido & Un sitio o aplicación \\
\hline Compuesto & Proveedor de contenido o ISP & Varios servicios de distinto tipo \\
Categoría de sitios & Proveedor de contenido o ISP & Varios servicios del mismo tipo \\
\hline Falso & Indirecto (mediante publicidad) & Sin restricción \\
\hline
\end{tabular}

Fuente: Elaboración propia.

(antes denominado Internet.org), creado y desarrollado por Facebook, que ofrece acceso gratuito a varias páginas web de distintos servicios (información, meteorología, etcétera) en países con bajo nivel de penetración de internet.

- Categoría de sitios o categórico (categorical zero-rating): Se asemeja a la tasa cero compuesta en que ofrece acceso gratuito a varias aplicaciones, pero a diferencia de ella, en el zero-rating categórico las aplicaciones ofrecidas pertenecen a una misma categoría de servicios (Keenan-Alspector, 2016: 9-10). Dos ejemplos de zero-rating de categoría de servicios son los programas Music Freedom y Binge On, ambos ofrecidos por la compañía T-Mobile, que permiten a sus clientes acceder sin consumo de datos a diferentes sitios y aplicaciones de streaming de música (como Spotify, Apple Music, Tidal y Soundcloud) y video (como Youtube, Netflix y Hulu), respectivamente.

- Falso: De acuerdo con la literatura académica, es una práctica que tiene la apariencia de tasa cero, pero que no lo es. En este modelo, un proveedor de contenido se asocia con un ISP para ofrecer cantidades limitadas de datos gratuitos a los usuarios a cambio de que éstos cumplan con ciertas condiciones, como ver alguna publicidad o descargar una aplicación. Los usuarios tienen la ventaja de utilizar el crédito de datos en la forma que ellos deseen, incluidos los servicios que no pertenecen al proveedor de contenidos que patrocina los datos (Carrillo, 2016: 381).

Dado que los avances tecnológicos y la innovación de las prácticas comerciales pueden sumar variables a los modelos expuestos, y con ello derivar en nuevos modelos, las prácticas antes enumeradas no pretenden ser una muestra comprensiva del zero-rating, sino que permiten enfocar nuestro estudio en los modelos más típicos que existen en Chile y el resto del mundo. 


\section{Calificación jurídica y regulación}

A partir de las teorías de la neutralidad de la red en general, y los análisis del zerorating en particular, podemos determinar tres posibles teorías sobre la calificación jurídica de la tasa cero respecto de la neutralidad de la red.

En primer lugar, están los autores que consideran que el zero-rating debe ser prohibido de modo absoluto por constituir una trasgresión per se a la neutralidad de la red, dado que ese principio busca el trato igualitario del tráfico de internet, y la tasa cero ejerce una discriminación tarifaria entre contenidos. Para algunos activistas de la neutralidad de la red, esto va aparejado a una crítica a los data caps, ya que constituyen un tipo de escasez artificial que finalmente incide en el comportamiento de los usuarios en internet (Layton y Elaluf-Calderwood, 2015: 3; Rossini y Moore, 2015: 5-6).

Otros autores plantean que el zero-rating no debe ser prohibido por la neutralidad de la red (Layton y Elaluf-Calderwood, 2015: 4-6). Incluso hay quienes afirman que la discusión sobre la tasa cero es meramente semántica, ya que esta práctica no tiene efecto en la habilidad de los usuarios de elegir los contenidos a los que acceden en la red y, por tanto, no vulnera la neutralidad de la red. ${ }^{8}$ También hay quienes avalan esta práctica por ser económicamente beneficiosa:

Los programas de zero-rating que se observan en el mercado se explican fácilmente como mecanismos impulsados por éste para capturar las eficiencias económicas asociadas con las características de los mercados de tecnología de la información. Al expandir el alcance del contenido y los servicios de distribución en línea, generan beneficios socioeconómicos. Las preocupaciones de que el zero-rating pueda servir como un medio para excluir la competencia o limitar la libertad de expresión parecen estar fuera de lugar y carecen de soporte tanto teórico como empírico (Eisenach, 2015: 9).

Sin embargo, existen autores (Jordan y Ghosh, 2010; Carrillo, 2016) que coinciden en que la discusión sobre la neutralidad en la red no puede darse en términos absolutos, mediante la prohibición o aceptación total de una práctica, como lo hacen las teorías anteriores. Por ello, según estas teorías, dependerá del análisis de cada modelo si el zero-rating atenta o no contra la neutralidad de la red.

Jordan y Ghosh (2010) plantean un método para establecer si una práctica de manejo del tráfico de internet está en conformidad con la neutralidad. Para ello recogen los conceptos de prácticas «razonables» $y$ «no razonables», que son utilizados desde el inicio del debate de la neutralidad de la red, y que incluso fue uno de los tres

8. David Post, «Facebook, Internet.org and the net neutrality bugaboo», The Washington Post, 17 de agosto de 2015, disponible en https://wapo.st/2M9vBvJ. 
principios centrales (transparencia, no bloqueo y antidiscriminación no razonable) de la Internet Open Order, primer instrumento que consagró la neutralidad de la red en los Estados Unidos (Mois, 2014: 147). La determinación de lo que se considera o no razonable es realizada por Jordan y Ghosh a partir de las respuestas a un test de cuatro criterios: i) dónde, o la capa en que se opera (transporte, red o datos); ii) qué, o la funcionalidad que afectan; iii) quién, o el sujeto que realiza la operación; y iv) cuándo, o la forma y propósito en los que se sustenta dicha práctica; los dos primeros atienden a las técnicas de gestión de tráfico, y los dos últimos a las prácticas de gestión de tráfico. Si alguna de las respuestas a estas preguntas no es razonable, habría razón suficiente para al menos cuestionar la gestión del tráfico del ISP por atentar contra la neutralidad de la red.

Si bien el caso del zero-rating no está entre las prácticas analizadas por Jordan y Ghosh, es posible aplicarle el test de razonabilidad. En todos los modelos de tasa cero, las preguntas dónde y qué serían fácilmente superadas, pues la diferenciación entre servicios no es técnica, sino tarifaria. En la pregunta quién, la tasa cero podría calificarse como razonable siempre que sea iniciativa de un ISP en respuesta al interés de los usuarios; en cambio, podría existir una alerta de afectación a la neutralidad si la práctica responde al patrocinio del proveedor del contenido. En la pregunta cuándo, se debe analizar qué tan razonablemente discriminatoria resulta la diferenciación de precio, lo cual puede determinarse, por ejemplo, a partir de la cantidad y tipos de servicios priorizados.

Otra teoría de este tipo es la desarrollada por Carrillo (2016), quien considera a la neutralidad de la red como parte del derecho internacional de los derechos humanos, por lo que afirma que los análisis meramente técnicos no son suficientes. Por ello, analiza el zero-rating desde tres criterios jurídicos generales: i) razonabilidad u objetivo legítimo de la práctica; ii) necesidad, lo cual depende del contexto; y iii) proporcionalidad, que variará según el tipo de zero-rating, y de los beneficios y consecuencias negativas de su aplicación. Así, la aplicación del zero-rating podría considerarse legítima, necesaria y proporcional en un contexto en que el costo de acceder a internet sea prohibitivo, como ocurre en países bajo el umbral de desarrollo. Por el contrario, en países desarrollados, la tasa cero podría no cumplir con dichos criterios, por lo que sería razonable su prohibición o limitación. La teoría de Carrillo puede complementar a la de Jordan y Ghosh, ya que aporta guías para responder a la pregunta cuándo, relativa al propósito de la práctica.

\section{La regulación del zero-rating}

La práctica del zero-rating puede ser abordada de diferentes maneras, como lo plantea Soares Ramos (2014: 18), quien formula cuatro modelos de regulación de la tasa cero: 
- Prohibición (total) de manera expresa o tácita. ${ }^{9}$

- Prohibición (atenuada), que acepta únicamente como excepción a las aplicaciones de utilidad pública.

- Autorización (parcial), que acepta los planes que extienden la gratuidad a todas las aplicaciones de un tipo de servicio determinado.

- Autorización (total), ya sea por la inexistencia de legislación específica, o por una regulación que la permite expresamente en todos los casos.

Considerando que la variedad de modelos que puede adquirir la tasa cero hace extremadamente compleja su calificación en términos absolutos - ya sea como prohibición o autorización total-, pensamos que una aproximación intermedia de la legislación resulta sensata. Una forma de realizar aquello es mediante la autorización o prohibición específica de los modelos de zero-rating que analizamos anteriormente.

Adherimos a la opinión de Keenan-Alspector (2016), que descarta de plano a las variedades categórica y falsa de zero-rating como atentatorias contra la neutralidad de la red, debido a que en ninguno hay una discriminación entre contenidos: en el primero, porque los usuarios utilizan sus créditos de datos en los contenidos que eligen, mientras que en el segundo porque, si bien se hace una diferenciación tarifaria, ésta se extiende a todos los sitios o aplicaciones de un mismo tipo de servicio, sin dar prioridad a ningún sitio en específico y dejando al usuario la libertad de elegir cuál utilizar.

Esto dejaría a los restantes tipos de zero-rating como atentatorios contra la neutralidad de la red, ya que en ellos el ISP o el proveedor de los contenidos inciden directamente en la elección de los usuarios finales. Según el test de Jordan y Ghosh, éstas serían prácticas no razonables, ya sea porque el patrocinio de los datos corre por cuenta del proveedor del contenido, como en el modelo de datos patrocinados, o porque se realiza una discriminación tarifaria no razonable que prioriza un tipo de servicio por sobre los demás, como en los zero-rating de un sitio o de un conjunto seleccionado de sitios (zero-rating compuesto).

Asimismo, recogemos las objeciones a las reglas de discriminación basadas en el caso a caso, ya que pueden causar incerteza en la industria y sumar un nuevo factor de discriminación en la adjudicación a posteriori (Van Schewick, 2010: 6). Por eso, es de nuestra opinión que la determinación de las prácticas de tasa cero prohibidas y permitidas debe ser establecida ex ante por la normativa, lo que descarta de plano a la desregulación como la solución viable, pero deja espacio para las excepciones a la aplicación estricta de la neutralidad de la red.

9. De acuerdo con la OCDE (2016: 81), han seguido este modelo las regulaciones de Canadá, Chile, Noruega, Países Bajos y Eslovenia. 
Las distintas legislaciones no solo pueden seguir distintos modelos en cuanto al fondo, es decir, a las prácticas permitidas o prohibidas por la neutralidad de la red, sino que también a la forma en que se hace cumplir la normativa. Entre los modelos que puede seguir la observancia de la neutralidad de la red están (Marsden, 2016: 35):

- Regulador de las telecomunicaciones: Un organismo administrativo vigila y sanciona el cumplimiento de las normas de neutralidad por los ISP. Tiene como problema que su ejecución puede variar según la motivación del regulador de turno.

- Parámetros regionales: Establecer una respuesta coordinada por las agencias a nivel regional (como el Organismo de Reguladores Europeos de Comunicaciones Electrónicas). Tiene la complejidad de que, si no se cuenta con una institucionalidad internacional fuerte, puede convertirse en una mera declaración de intenciones sin aplicación práctica.

- Derecho de la competencia: Los promotores de este último enfoque por lo general hacen la distinción entre la discriminación técnica, que debería ser entregada al regulador de las telecomunicaciones, y la discriminación comercial entre proveedores de contenidos, que en su opinión es materia de libre competencia. Dentro de los criterios para establecer al zero-rating como una práctica de discriminación comercial están: i) las aplicaciones beneficiadas son por lo general las predominantes; ii) los ISP no velan por la diversidad de los contenidos en internet; iii) el zero-rating no impulsa el acceso a internet en mercados consolidados; $y$ iv) esta práctica fortalece las posiciones de mercado de los proveedores de contenido (Brouwer, 2017: 38).

Incluso dentro del análisis de la tasa cero como cuestión que debe ser estudiada por el derecho de la competencia, se replica el debate sobre la consideración del zerorating como una práctica que debe permitirse o prohibirse. Por ejemplo, la Comisión Europea (2017: 134) ha concluido que la tasa cero tiene efectos anticompetitivos en los casos en que los ISP liberan solo sus propios contenidos o liberan los de terceros mediante acuerdos (patrocinados o no) que establecen una exclusividad, es decir, que impiden que se beneficie a los competidores de dicho proveedor. Discrepamos de esta posición, pues de todas formas daría lugar a la discriminación comercial de las plataformas, ya que puede que un ISP decida otorgar tasa cero a un solo servicio - por ejemplo, Facebook - dentro de una categoría, sin tener un contrato de exclusividad con dicho proveedor, lo cual además de ser atentatorio contra la neutralidad positiva, reforzaría la posición predominante de dicho servicio en el mercado.

Una posición mucho más coherente con ambas concepciones de la neutralidad de la red es la del regulador canadiense, la Comisión de Radiodifusión y Telecomunica- 
ciones de Canadá, ${ }^{10}$ cuya opinión sobre el zero-rating es negativa, pues a largo plazo «podría ampliar o consolidar las posiciones de mercado ya favorables de los grandes ISP y de los proveedores de contenidos, y podría impedir o limitar la actividad competitiva de actores más pequeños, lo que sería perjudicial para los consumidores». Al igual que en la neutralidad positiva, se prohíbe dar un trato preferente a un proveedor que por lo general ya tiene dicha posición en el mercado, y se propicia que haya un trato igualitario por parte del ISP.

Luego de haber analizado los diferentes modelos de regulación existentes en relación con la neutralidad de la red y el zero-rating, estudiaremos la legislación y el criterio del regulador en Chile, para determinar su correspondencia con los modelos antes señalados.

\section{El caso chileno: La Ley 20.453}

La Ley 20.453, que «Consagra el principio de neutralidad en la red para los consumidores y usuarios de internet», fue promulgada el 18 de agosto de 2010 y publicada en el Diario Oficial el 26 de agosto del mismo año. Consistió en una modificación de la Ley General de Telecomunicaciones, que agregó tres artículos relativos a la neutralidad de la red (24.h, 24.i y 24.j). De éstos, el primero es el que se analizará en profundidad para determinar las características de la regulación chilena de la neutralidad de la red.

\section{Análisis del artículo 24.h}

El artículo 24.h de la Ley 20.453 establece que las obligaciones sobre el respeto de la neutralidad en la red están dirigidas a los ISP, definidas como las «concesionarias de servicio público de telecomunicaciones que presten servicio a los proveedores de acceso a internet y también estos últimos; entendiéndose por tales, toda persona natural o jurídica que preste servicios comerciales de conectividad entre los usuarios o sus redes e Internet». ${ }^{11}$ Dichos proveedores de internet, de acuerdo con el literal a) de dicho artículo, «no podrán arbitrariamente bloquear, interferir, discriminar, entorpecer ni restringir el derecho de cualquier usuario de internet para utilizar, enviar, recibir u ofrecer cualquier contenido, aplicación o servicio legal a través de internet, así como cualquier otro tipo de actividad o uso legal realizado a través de la red» (éstos y los próximos destacados similares son nuestros). Los verbos rectores «bloquear», «interferir», «discriminar», «entorpecer» $\mathrm{y}$ «restringir» son la clave para determinar la extensión en la regulación de las actividades de gestión de tráfico en la legislación chilena.

10. «Telecom regulatory policy», Canadian Radio-television and Telecommunications Commission, CRTC 2017-104, 20 de abril de 2017, disponible en: https://bit.ly/2loEFW3.

11. El texto de la Ley 20.453 está disponible en http://bit.ly/2IiXPld. 
No cabe duda de que en los verbos «bloquear»y «restringir» existe una alusión directa a la neutralidad negativa, en el sentido de prohibir el acceso o el uso de ciertos servicios. Respecto de los verbos «interferir» $\mathrm{y}$ «entorpecer», se hace alusión a acciones que, si bien no implican un bloqueo de los servicios, sí perturban el ejercicio de los usuarios de internet sobre dichos servicios, por cuanto también pueden calificarse como un tipo atenuado de prácticas negativas que atentan contra la neutralidad de la red.

Ahora bien, el verbo «discriminar» es el menos pacífico, dado que deja lugar a dos interpretaciones: una interpretación restrictiva, en que «discriminar» se referiría única y exclusivamente a la neutralidad negativa, tal como los demás verbos rectores del artículo 24.h letra a; y una interpretación extensiva, en que incluiría tanto a la neutralidad negativa como positiva. Si se acogiera esta última tesis, podría dar lugar a la prohibición de las prácticas de zero-rating por la ley chilena.

La primera interpretación sería coherente con una exposición realizada por el subsecretario de Telecomunicaciones chileno en la cumbre de alto nivel que reunió a los reguladores de las telecomunicaciones de Europa, América Latina y el Norte de África, desarrollada en julio de 2015. ${ }^{12}$ En esa ocasión, se expusieron como los tres principios que establece la Ley 20.453: i) transparencia, ii) no bloqueo de contenidos, y iii) aplicaciones e indicadores de calidad. La principal obligación para los ISP, de acuerdo con estos principios, sería el no bloqueo de los contenidos, definido por el subsecretario Huichalaf (2015a: 10) como «el derecho de acceder libremente a cualquier tipo de contenido o servicio legal en internet, sin que el proveedor pueda negar dicho acceso». Llama la atención que dentro de los pilares de la ley solo se considere el «no bloqueo», lo que deja fuera a la «no discriminación» - a diferencia de la Internet Open Order de Estados Unidos-, ya que esto podría dar a entender que la ley solo consagra la neutralidad de la red en su sentido negativo, dado que el objetivo del cuerpo legal sería evitar que el ISP pueda negar dicho acceso.

Este razonamiento es el que han seguido las empresas telefónicas para continuar ofreciendo los programas zero-rating. De acuerdo con el gerente general de la compañía Virgin Mobile, «el principio de neutralidad de la red busca precisar que el servicio de internet no sea objeto de discriminaciones, de manera que no se obstaculicen aplicaciones y contenidos de manera arbitraria». ${ }^{13}$ Similar argumento dio un representante de la compañía WOM al afirmar que «no existe una violación a la normativa, pues no existe discriminación al servicio de datos, aplicaciones o contenidos», debido

12. La cumbre de alto nivel de Barcelona reunió a los reguladores nacionales agrupados en BEREC (Europa), EaPeReg (Europa del Este), REGULATEL (América Latina) y EMERG (Mediterráneo y Norte de África).

13. Comunicación de Juan Antonio Etcheverry a la Subtel, de fecha 14 de abril de 2014, contenida en el Oficio Ordinario 5.374. 
a que las ofertas de tasa cero «no afectan de manera alguna el libre acceso a cualquier página de internet, aplicaciones o contenidos, ya que con el saldo en el caso de prepago o con la cuota de navegación en el caso de pospago, los clientes podrán acceder a cualquier sitio sin restricción ni bloqueo por parte de WOM». ${ }^{14}$ En resumen, para las compañías telefónicas, la diferenciación tarifaria de aplicaciones no sería un tipo de discriminación en términos de la ley, toda vez que el verbo rector «discriminar» está circunscrito al bloqueo o perturbación del acceso a dichos servicios.

Estamos plenamente convencidos de que la interpretación antes expuesta de la ley es inexacta, no solo porque el regulador se desdijo respecto de lo que consideraba como sus fundamentos, al cambiar el principio de «no bloqueo» por el de «neutralidad tecnológica», ${ }^{15}$ sino también porque no existe motivo que permita afirmar que la palabra «discriminar» en la ley haga referencia únicamente a la discriminación negativa y no a la positiva, como han entendido las empresas telefónicas.

Lo anterior queda de manifiesto con el solo examen del inciso segundo del artículo 24.h letra a), que establece que:

[Los ISP] deberán ofrecer a cada usuario un servicio de acceso a internet o de conectividad al proveedor de acceso a internet, según corresponda, que no distinga arbitrariamente contenidos, aplicaciones o servicios, basados en la fuente de origen o propiedad de éstos, habida cuenta de las distintas configuraciones de la conexión a internet según el contrato vigente con los usuarios.

La norma utiliza un verbo sinónimo de discriminar («distinguir»), pero no se hace referencia a la naturaleza negativa o positiva de dicha distinción, sino que solo se afirma que los «contenidos, aplicaciones o servicios» no deben ser discriminados arbitrariamente con base «en la fuente de origen o propiedad de éstos».

Dado que los verbos rectores del artículo 24.h de la Ley 20.453 son definidos en términos amplios —opinión compartida por Castro, Pereira da Silva y Viollier (2017: 25) -, debemos acudir a otras fuentes del derecho para demostrar que la normativa chilena consagra el principio de neutralidad de la red en sus acepciones negativa y positiva.

\footnotetext{
14. Raúl Estrada, «Pokémon Go, música ilimitada y redes sociales "gratis": ¿Se cumple la Ley de Neutralidad en la Red en Chile?», Fayerwayer, 26 de septiembre de 2016, disponible en https://bit.ly/2qPSQm9.

15. Durante otra presentación de la Subtel en la cumbre de reguladores de las telecomunicaciones de 2015, Huichalaf (2015b) planteó como principios de la ley: i) potestad a la Subsecretaría de Telecomunicaciones; ii) neutralidad tecnológica; iii) gestión y administración de tráfico; y iv) transparencia. De este modo, tampoco habría razón desde el regulador chileno para plantear que el ámbito de la neutralidad protegido por el cuerpo legal se ciñe solamente al «no bloqueo», sino que protege a la neutralidad de la red en su amplio alcance.
} 


\section{El espíritu de la ley}

La ley de neutralidad de la red fue aprobada sin oposición; primero en el Senado - entonces compuesto por 38 escaños-, por 30 votos a favor, ninguno en contra y una abstención, ${ }^{16}$ y posteriormente en la Cámara de Diputados, por 99 votos a favor, ningún voto en contra y una abstención, de un total de 120 escaños. ${ }^{17}$ Ello demuestra el amplio consenso que obtuvo el proyecto de ley en todo el espectro político, incluyendo el apoyo de los Gobiernos de Michelle Bachelet y Sebastián Piñera.

Por ello, la historia de la discusión parlamentaria que dio origen a la Ley 20.453 se constituye como una fuente esencial a la hora de estudiar cuál fue la voluntad del legislador en su promulgación — siguiendo al artículo 19 inciso segundo del Código Civil chileno- y junto con ello verificar si se recogen ambos conceptos de la neutralidad de la red.

La moción parlamentaria que dio origen a este proyecto de ley, presentada en marzo de 2007 por diez diputados de las dos principales coaliciones políticas del país (Alianza y Concertación), justificaba la necesidad de reconocer la neutralidad de la red por las prácticas de los ISP «que buscan en definitiva "condicionar" el acceso de los usuarios de internet, discriminando contenidos, aplicaciones o dispositivos». En ella, establecen que uno de los objetivos a proteger es la innovación, «pues las páginas web, los servicios y aplicaciones de la [red] son exitosas o fracasan por su propio mérito, lo cual es determinado por la decisión diaria de millones de usuarios». ${ }^{18}$

En la misma moción parlamentaria, se explica que la neutralidad de la red «consiste en que ésta en sí misma (no sus extremos) transmita toda información sin mirar ni jerarquizar y tampoco priorizar, con lo cual se asegura que la red es la misma para todos». Los verbos utilizados invocan tanto a la neutralidad negativa («jerarquizar») como positiva («priorizar»). Con ello, agregan, busca evitarse que el mercado derive en el modelo de jardines vallados:

Sin la «neutralidad de la red», internet se parecería más a un canal de cable, pues los controladores de la red decidirían qué contenidos, aplicaciones o páginas se pueden ver a partir de criterios propios inconsultos y los usuarios solo se limitarían por tanto a escoger de un menú que su ISP le quiere proporcionar, generalmente condicionado por múltiples intereses económicos de toda índole, con lo cual se pierde una de las grandes ventajas y beneficios de una red libre, guiada exclusivamente por la preferencia de los usuarios. ${ }^{19}$

16. «Historia de la Ley 20.453», Biblioteca del Congreso Nacional, 20 de agosto de 2010, folio 147, disponible en http://bit.ly/2IhmoPI.

17. «Historia de la Ley 20.453», folio 166.

18. «Historia de la Ley 20.453», folio 4.

19. «Historia de la Ley 20.453», folio 5. 
Durante la discusión del proyecto en el Congreso Nacional, se continuó reforzando la idea de que el objeto de la neutralidad era evitar las conductas de discriminación, tanto positivas como negativas. El diputado Marcelo Díaz, uno de los autores de la moción parlamentaria, explicó en la sesión 89 del 11 de octubre de 2007:

El principio básico de la iniciativa en discusión, que, por cierto, debemos compartir y respaldar, se orienta a que el usuario o consumidor contrata el acceso a internet de manera libre para navegar, también de manera libre, por el denominado ciberespacio, y no para que algún gran proveedor o suministrador lo oriente explícita $o$ implícitamente respecto de determinados contenidos. ${ }^{20}$

Díaz planteó que los proveedores de servicios de internet no pueden «orientar» al usuario a que utilice una determinada plataforma de forma explícita o implícita, dado que ello afecta su libertad para navegar por internet. Podemos entender que las prácticas que regula la neutralidad positiva - como el zero-rating-, son una forma en que el proveedor de internet orienta de manera implícita a los consumidores para utilizar un servicio, ya que se ofrece como incentivo no cobrar por los datos utilizados.

El entonces senador Carlos Ominami, en la sesión 16 del 30 de abril de 2008, hizo referencia al alcance del verbo discriminar, al definirlo como «todo cuanto signifique evitar que puedan establecerse restricciones, privilegiar determinados contenidos o proveedores de ellos, incluso bloqueando ciertas prestaciones, [lo cual] claramente tiene que ser prohibido». ${ }^{21}$ Ominami dice que la discriminación tiene como fin tanto la restricción (neutralidad negativa) como el privilegio (neutralidad positiva) de ciertos servicios por sobre otros. Además, agrega que dicha conducta puede ser realizada «incluso» mediante el bloqueo, con lo cual se reafirma que las acciones negativas no son las únicas que pueden afectar a la neutralidad de la red, sino que dicha conducta es la más extrema que podría realizar un ISP.

En la sesión 51 del 13 de julio de 2010, la diputada Marcela Sabat hizo mención explícita a los dos tipos de neutralidad: la negativa, al afirmar que «las prácticas detectadas de ciertas compañías que tienen como objetivo limitar la libertad de los usuarios, a fin de priorizar ciertos contenidos respecto de otros», y la positiva, al plantear que los ISP pueden «favorecer a determinados servidores, los que, a su vez, están dispuestos a pagar por ello».2 Esta última descripción de Sabat es concordante con el zero-rating, que favorece a ciertos contenidos respecto de otros, y agregó que la práctica discriminatoria puede incluso ser patrocinada por el proveedor de los servicios favorecidos, como ocurre en el modelo de datos patrocinados.

20. «Historia de la Ley 20.453», folio 25 .

21. «Historia de la Ley 20.453», folio 89.

22. «Historia de la Ley 20.453», folio 156 . 
En la misma sesión, el diputado Gonzalo Arenas, quien también fue promotor del proyecto de ley, quiso establecer expresamente a qué se refería la ley con la arbitrariedad de las acciones, de forma de evitar interpretaciones erróneas. ${ }^{23}$ Arenas planteó "que el concepto "arbitrario" se va a dar en el contexto de que se va a permitir que haya una gestión razonable de la red, pero que esa gestión sea igualmente aplicable para todos». ${ }^{24}$ Cabe destacar la coincidencia de esta interpretación con los conceptos de prácticas «razonables» $\mathrm{y}$ «no razonables» planteada por el test de Jordan y Ghosh.

En definitiva, el análisis de la historia de la Ley 20.453 nos permite concluir que el espíritu de la legislación es la protección de la neutralidad de la red en su amplio concepto, que impide no solo las acciones negativas (bloqueo, entorpecimiento), sino también las que promueven o benefician a ciertos servicios por sobre otros, con lo que se descartan de este modo las interpretaciones empleadas por las empresas telefónicas para promover el zero-rating.

\section{La aplicación de la ley}

Tras haber analizado la ley chilena, que cautela la neutralidad positiva, en esta sección estudiaremos cuáles han sido los criterios del organismo regulador (la Subsecretaría de Telecomunicaciones) para aplicarla, específicamente respecto de la tasa cero, a partir del análisis del reglamento de la ley y de la práctica. Con ello determinaremos los motivos de la tolerancia del zero-rating por las empresas telefónicas chilenas.

\section{El reglamento de la ley}

El reglamento de la ley de neutralidad de la red está contenido en el Decreto 368, dictado por la Subtel en diciembre de 2010 y publicado el 18 de marzo de 2011. Un reglamento es una norma emanada de un órgano de la Administración del Estado que complementa lo que se establece en la norma legal.

La dictación del Decreto 368 tuvo la particularidad de haber sido fuertemente inspeccionada por las organizaciones de la sociedad civil que promueven la neutralidad de la red, por lo que su versión inicial fue criticada por la carencia «de herramientas de control y [porque] no representaba el espíritu de la ley», lo cual fue corregido, según el organismo regulador (Huichalaf, 2015a: 16). Con estos antecedentes, se hace necesario examinar el texto del reglamento, con el objeto de determinar el criterio que el regulador tuvo inicialmente respecto de la aplicación de la neutralidad de la red y contrastarlo con el espíritu de la ley, que supuestamente representa.

23. Como ejemplo de interpretación errónea, podemos citar la definición de arbitrariedad con que la compañía VTR justificó que sus ofertas de redes sociales gratis no trasgredían la ley: «Acto o proceder contrario a la justicia, la razón o las leyes, dictado por la sola voluntad o el capricho» (Oficio Ordinario 5.075).

24. «Historia de la Ley 20.453», folio 164. 
En el literal c) del preámbulo del decreto se define el objetivo de la legislación de neutralidad de la red, al establecer que «la Subsecretaría debe velar para que los servicios, aplicaciones y contenidos de internet sean ofrecidos sin discriminación, a la vez que el acceso por parte de los usuarios a ellos sea permitido sin restricciones arbitrarias por parte de los concesionarios o ISP $»{ }^{25}$ Dicho texto hace referencia a las formas en que la neutralidad de la red puede verse afectada, ya sea por una discriminación en la oferta (positiva o negativa), como por «restricciones arbitrarias» (negativa).

Esta concepción es desarrollada y explicitada en el artículo 8 del reglamento, donde se ejemplifican las prácticas restrictivas a la libertad de utilización de los contenidos, aplicaciones o servicios, mediante cuatro numerales, de los cuales los más relevantes para efectos de nuestro análisis son los números 1 y 2 . En el primero se establece que:

Toda aquella acción que, arbitrariamente, tienda a bloquear, interferir, entorpecer, restringir y/o de cualquier forma obstaculizar el derecho de cualquier usuario de internet para utilizar, enviar, recibir u ofrecer cualquier contenido, aplicación o servicio legal a través de internet, así como cualquier otro tipo de actividad o uso legal realizado a través de la red, en especial, aquellas medidas de gestión de tráfico o administración de red que, en aquel carácter, afecten a los niveles de servicio contratados por el respectivo usuario.

El reglamento agrupa en el numeral 1 a todos los verbos rectores del artículo 24.h de la ley, con la sola excepción de «discriminar». Todos ellos («bloquear», «interferir», «entorpecer» $\mathrm{y}$ «restringir») se caracterizan por ser acciones negativas que atentan contra la neutralidad. A ellos agrega «obstaculizar», lo cual refuerza la descripción de conductas negativas.

El verbo rector «discriminar» es introducido en el numeral 2, donde se utiliza en un carácter exclusivamente positivo, al establecerlo como sinónimo de «priorizar», verbo que no fue incluido en la Ley 20.453:

Toda aquella acción que, arbitrariamente, tienda a priorizar o discriminar entre proveedores de contenidos, aplicaciones y/o usuarios. En todo caso, siempre se entenderá como arbitraria la acción de priorización o discriminación que afecte a proveedores de contenidos, aplicaciones y/o usuarios respecto de otros de similar naturaleza.

En ambos numerales, además, se reitera el concepto de arbitrariedad establecido en la ley. El regulador innova al introducir una definición de aquello que «siempre»se

25. «Decreto 368, Reglamento que regula las características y condiciones de la neutralidad de la red en el servicio de acceso a internet», Biblioteca del Congreso Nacional, 18 de marzo de 2011, disponible en http://bit.ly/2IgGY2C. 
considerará arbitrario en el numeral 2, esto es, cuando afecte a proveedores de contenidos, aplicaciones o usuarios respecto de otros de similar naturaleza. Es decir, según el reglamento no se permite, en ningún caso, una discriminación (priorización) a aplicaciones y usuarios de igual naturaleza. En esto se sigue el espíritu de la ley, que considera la arbitrariedad como el hecho de que una gestión razonable del ISP sea igualmente aplicada para todos, aunque con un leve matiz, pues interpreta la palabra «todos» como «todos aquéllos de la misma naturaleza».

Ahora bien, eventualmente se podría establecer, a contrario sensu, que el regulador acepta la discriminación (priorización) de servicios, siempre y cuando ésta se extienda a todas las aplicaciones «de similar naturaleza», por lo que inserta una excepción a la neutralidad positiva. En el caso del zero-rating, se permitiría solo la aplicación de esta práctica a todas las aplicaciones de un mismo tipo (zero-rating categórico), lo cual es coherente con la noción de que esta práctica no representa mayor amenaza a la neutralidad de la red. Sin embargo, ninguna de las ofertas de tasa cero vigentes en Chile tiene esta característica, a pesar de que se las engloba como «redes sociales gratis»; esto es corroborado por los oficios de la Subtel que han llamado a adaptar sus promociones a la normativa vigente (véase anexo 1). Para acogerse a esta excepción, las empresas telefónicas deberían extender su oferta a las demás aplicaciones de «similar» naturaleza. ${ }^{26}$

En síntesis, los criterios plasmados por el regulador en el reglamento de la ley son: i) se reconoce expresamente que el espíritu de la ley busca la prohibición de las conductas tanto positivas como negativas que atentan contra la neutralidad, lo cual incluye al zero-rating; y ii) se interpreta la arbitrariedad de las conductas discriminatorias como la no aplicación de dicha diferenciación a las demás aplicaciones de igual naturaleza.

\section{El criterio práctico de la Subtel}

La Subtel está mandatada por la ley de neutralidad de la red a velar por su cumplimiento y ejercer las sanciones administrativas a los ISP por las infracciones a las obligaciones legales o reglamentarias que éstos cometan, según lo establecido por el artículo 24.i. Su rol es fundamental, pues la experiencia comparada demuestra que no basta con tener una normativa que prohíba este tipo de prácticas, ya que, si el regulador es débil y no posee las competencias necesarias para hacer cumplir la ley, resulta

26. Dentro de las ofertas de las empresas de telefonía en Chile, se agrupan redes sociales propiamente tales (Facebook, Twitter e Instagram), aplicaciones de mensajería (Whatsapp, Facebook Messenger y Snapchat) e incluso servicios de streaming de música (Spotify, iTunes). Todas estas son «similares» en el sentido de que implican intercambio de contenidos entre usuarios, pero lógicamente no agotan ni la categoría general de «redes sociales», ni las categorías específicas de servicios antes mencionadas (por ejemplo, en mensajería restaría por incluir a Telegram, Signal y Line, entre otras). 
muy difícil que ésta se respete; la literatura ejemplifica dicho problema con el caso de Eslovenia (Marsden, 2016: 13).

En Chile, las ofertas de redes sociales gratis fueron introducidas en 2012 - bajo la plena vigencia de la ley y el reglamento de neutralidad- por dos nuevos operadores de telefonía móvil, Virgin Mobile y VTR, y progresivamente se extendió a las demás empresas existentes, debido a la alta competencia en el mercado favorecida por la portabilidad numérica, establecida por la Ley 20.471 de 2010. De este modo, el zero-rating se convirtió en una práctica estándar de las compañías para atraer nuevos clientes, con un enfoque en los servicios más populares, como Facebook, Instagram, Twitter o Whatsapp. No hay indicios de que la adopción de la tasa cero haya ido aparejada de acuerdos entre las empresas de telefonía y las proveedoras de los contenidos priorizados, por lo que no se trataría de los modelos de zero-rating compuesto o de datos patrocinados, sino que más bien del modelo de tasa cero de un único servicio, establecido por iniciativa y patrocinio de los ISP.

Durante los dos primeros años en que funcionaron las ofertas de redes sociales gratis no hubo anuncios públicos de la Subtel al respecto, aunque se conocen las comunicaciones privadas que el organismo mantuvo con Neutralidad Sí, una organización de la sociedad civil que había denunciado a la compañía Virgin Mobile por sus prácticas de zero-rating. En ese intercambio, un abogado de la Subtel afirmó que la tasa cero no afectaba a la neutralidad de la red, ya que no constituía un bloqueo del acceso a internet, lo que reducía la neutralidad a su concepto negativo. ${ }^{27}$

Dicho criterio temprano - y desde luego erróneo- fue desechado completamente con el Oficio Circular 40 dictado por la Subtel el 14 de abril de $2014,{ }^{28}$ el cual determinó que las ofertas de redes sociales gratis eran una contravención a las normas de la neutralidad de la red, particularmente a su artículo 24.h, letra a).

La referida norma no solo tiene por finalidad evitar el «bloqueo», sino también, y según lo señalan los otros verbos rectores que lo componen, prohibir toda «discriminación», «interferencia» o «restricción» de contenidos, aplicaciones o servicios, por ende dispone con claridad que los concesionarios de servicios públicos de telecomunicaciones que provean de servicios de acceso a internet y los ISP, no podrán discriminar en el derecho de los usuarios a recibir u ofrecer cualquier contenido, aplicación o servicio.

Este criterio fue confirmado por la Subtel en oficios dirigidos a las empresas telefónicas Claro, VTR Wireless, Telefónica Móviles (Movistar) y Virgin Mobile durante junio de $2014{ }^{29}$

27. Pepe Huerta, «Redes sociales gratis y la circular de Subtel: ¿Dónde surgió el problema?», Neutralidad Sí, 2 de junio de 2014, disponible en https://bit.ly/1GtoFTc.

28. El texto del Oficio 40 está disponible en https://bit.ly/2g5Sodt.

29. Oficios Ordinarios 5.074, 5.075, 5.084 (4 de junio de 2014) y 5.374 (11 de junio de 2014). 
La interpretación plasmada por el regulador en el oficio es coherente con el espíritu de la ley y el reglamento, pues identifica a las prácticas discriminatorias positivas como atentatorias a la neutralidad de la red. Asimismo, el oficio confirmó la interpretación del concepto de arbitrariedad realizada en el reglamento, al exigir a las empresas telefónicas la regularización de la práctica del zero-rating mediante su extensión a «las restantes aplicaciones disponibles que son de similar naturaleza o competencia».

Sin embargo, las declaraciones del subsecretario de Telecomunicaciones a distintos medios de comunicación causaron confusión, pues se calificaba al zero-rating como ilegal en todos los casos, incluso en los que no era arbitrario, lo que contravenía lo establecido en el reglamento y en la propia circular. Dicho criterio fue ampliamente replicado por medios digitales internacionales, lo cual contribuyó a difundir la concepción errónea de que la Subtel prohibía totalmente las ofertas de tasa cero. ${ }^{30}$

Alertada por dichas noticias, la Fundación Wikimedia, organización sin fines de lucro promotora de proyectos de conocimiento libre como la enciclopedia Wikipedia, contactó a la Subtel para consultar si la prohibición de las aplicaciones de tasa cero se extendía a Wikipedia Zero. ${ }^{31}$ La Subtel afirmó, en comunicación privada con esa fundación, que Wikipedia Zero podría funcionar en Chile, ya que no estaba vulnerando la ley de neutralidad de la red, y además aclaró que el oficio tenía como objetivo prohibir las prácticas en las que se «ofrecían paquetes que otorgaban acceso libre de costo de datos (zero-rated) a ciertas redes sociales, junto a planes de voz y de datos, y que la circular no pretendía tener una aplicación generalizada, o aplicada a otros casos diferentes», ${ }^{32}$ aunque no se explicó cuál era el fundamento para adoptar esa interpretación del oficio.

El criterio adoptado por la Subtel entre 2010 y 2014 respecto de la práctica del zerorating, que podía calificarse como armónico con la ley, el reglamento y el oficio, fue cambiado radicalmente durante la cumbre de alto nivel de reguladores realizada en junio de 2015, cuando redujo sus estándares para la autorización de las redes sociales gratis:

Es posible que se ofrezca acceso a algunas redes sociales gratuitas, o sea, que no se descuente de la cuota de tráfico para los pospago o bolsas y que no se descuente del saldo para los prepagos, en la medida que, conjuntamente con acceder a dichas redes sociales, se tenga también acceso conjuntamente a todo el resto de contenidos y a otras redes sociales no elegidas arbitrariamente por el operador y otras aplicaciones de internet (Huichalaf, 2015a: 23).

30. Leo Mirani, «When net neutrality backfires: Chile just killed free access to Wikipedia and Facebook», Quartz, 30 de mayo de 2014, disponible en https://qz.com/215064.

31. Para transparentar mi posición, cabe señalar que fui parte de esa comunicación en mi calidad de director de la Corporación Wikimedia Chile, capítulo local de la Fundación Wikimedia.

32. Yana Welinder y Carolynne Schloeder, «Ente regulador chileno le da la bienvenida a Wikipedia Zero», Wikimedia Blog, 23 de septiembre de 2014, disponible en http://bit.ly/2IipW3U. 
De este modo, se descartó la definición de arbitrariedad establecida por el reglamento, entendida como la discriminación - en este caso, gratuidad - respecto de ciertos tipos de servicios dentro de una misma categoría, toda vez que se permite que los ISP solo tengan planes de tasa cero para algunas redes sociales.

El nuevo criterio de la Subtel interpreta el concepto de arbitrariedad de las acciones discriminatorias como la no restricción al acceso conjunto de los demás servicios de igual naturaleza (redes sociales). Con esto se acepta tácitamente la tasa cero en todos los casos, ya que la única condición para las empresas telefónicas sería cumplir con la neutralidad negativa, es decir, no bloquear el acceso a los demás proveedores de servicios similares. Dicho estándar fue ratificado por el subsecretario Huichalaf en 2016, cuando afirmó que «si una persona tiene un plan de internet y la compañía le ofrece datos sin "costo" en una aplicación o en una página, nosotros creemos que aquello no viola la neutralidad de la red».33

Sin embargo, llama poderosamente la atención que el nuevo criterio instaurado por subsecretario Huichalaf no fuera seguido por su subalterno, el jefe de la División de Fiscalización, pues en tres oficios firmados por éste - una de ellas ejerciendo como subsecretario subrogante- se repitió el criterio del oficio Circular 40 de $2014 .^{34}$

Sumado a lo anterior, el regulador chileno decidió desligarse de la facultad de aplicar sanciones a las compañías por tener ofertas de zero-rating que no se adecúen a la ley, al afirmar que las prácticas que afecten a los proveedores de servicios o de internet debían ser revisadas por las autoridades competentes del derecho de la competencia:

La ley de neutralidad en la red no solo consagra el acceso sin distinciones a los usuarios, sino que también lo hace por el lado de quienes ofrecen servicios sobre internet, ya que si un operador les da condiciones de difusión y acceso especial a algunas y no a todas, en que los usuarios no pueden elegir, estaríamos en presencia de una conducta que afectaría la libre competencia y estas empresas se verían perjudicadas y discriminadas, es decir, sin igualdad de acceso a los usuarios y viceversa, lo cual cambia cuando los usuarios tienen acceso irrestricto a los contenidos y sus proveedores en igualdad de condiciones (Huichalaf, 2015a: 23).

Este criterio fue refrendado por el organismo en al menos dos oportunidades, durante una entrevista realizada al subsecretario Huichalaf en $2016,{ }^{35}$ y en la audien-

33. Raúl Estrada, «Pokémon Go, música ilimitada...».

34. Oficios Ordinarios 14.725 (12 de noviembre de 2015), 7.502 y 7.503 (16 de agosto de 2016).

35. El subsecretario de Telecomunicaciones Pedro Huichalaf afirmó: «¿Quién se siente discriminado con una oferta así? No el cliente, sino la empresa que se queda fuera del paquete. En la Subtel solo velamos por el lado del usuario y si éste tiene un problema para acceder a internet, entonces nos metemos. Pero si una empresa tiene un problema, debe ir al Tribunal de Libre Competencia» (Raúl Estrada, «Pokémon Go, música ilimitada...»). 
cia que funcionarios de la Subtel otorgaron a los investigadores de ONG Derechos Digitales (Castro, Pereira da Silva y Viollier, 2017: 27), ya durante la gestión del subsecretario Rodrigo Ramírez.

Bajo esta interpretación, el regulador se encargaría solo de asegurar el acceso irrestricto de los usuarios a la red, es decir, sin bloqueos ni interferencias, pero no del igual acceso a los contenidos, pues supuestamente esto sería un problema entre las empresas proveedoras de internet y de los contenidos, que correspondería analizar en sede de libre competencia. En definitiva, recoge la tendencia de división de la regulación entre la discriminación técnica, que recaería en la Subtel, y la discriminación comercial, que debería ser revisada por la Fiscalía Nacional Económica y el Tribunal de Defensa de la Libre Competencia.

Es de nuestra opinión que la decisión de la Subtel es del todo antojadiza por varios motivos. En primer lugar, porque intenta introducir una división artificial en los efectos que tiene la neutralidad de la red respecto de los tres sujetos involucrados (ISP, usuarios y prestadores de contenidos). Según Manuel Abarca, ${ }^{36}$ dicha interpretación impediría una regulación efectiva de la tasa cero, ya que «genera los problemas propios de cada ámbito de regulación, impidiendo hacer efectiva la opción institucional por la no discriminación». Esto se hace patente en la inexistencia de una política conjunta de las instituciones de libre competencia y derecho del consumidor respecto del zero-rating.

Cabe señalar también que el actual criterio de la Subtel se opone al que tenía el organismo durante la discusión de la Ley 20.453, cuando consideraba que uno de los objetivos de la regulación era precisamente evitar que la neutralidad de la red fuera considerada una materia de libre competencia, toda vez que dicho sistema sería poco eficaz en una legislación que, como la chilena, forma parte de la tradición jurídica continental. ${ }^{37}$

El segundo argumento para estar en contra de dicha interpretación es la carencia de un sustento legal para hacer esa diferencia en las facultades de la Subtel. Según funcionarios de la Subsecretaría, la justificación estaría en que no habría consenso respecto de sus facultades sobre «los ámbitos relacionados con el contenido mismo ni de la competencia entre los distintos actores», ya que estos últimos radicarían en la jurisprudencia del Tribunal de la Libre Competencia (Castro, Pereira da Silva y Viollier, 2017: 77). En palabras más sencillas: ante la duda, se abstienen.

36. Manuel Abarca, «Neutralidad de red y ofertas zero-rating», RegCom, 8 de noviembre de 2017, disponible en https://bit.ly/2LM7Jy8.

37. El subsecretario de Telecomunicaciones Pablo Bello afirmó en 2008 que «es importante regular esta materia porque el Tribunal de Defensa de la Libre Competencia no genera regulación ni sienta jurisprudencia, solo analiza caso a caso y lo que resolvió en un determinado caso no se transforma en un imperativo respecto de otros actores, a diferencia de lo que ocurre en el sistema anglosajón, que cuando se resuelve un tema que afecta a la libre competencia se genera una especie de regulación» («Historia de la Ley 20.453», folio 63). 
Si bien el Decreto Ley 1.762 define a la Subsecretaría como la «autoridad competente para conocer y resolver acerca de las materias de carácter técnico relativas a las telecomunicaciones» (artículo 7), lo cual podría sustentar la posición del regulador, también es cierto que dentro de sus funciones, establecidas por el artículo 6 del mismo decreto, está «velar por el cumplimiento de las leyes, reglamentos, normas técnicas y demás disposiciones internas», entre las que se cuenta la ley de neutralidad de la red y su reglamento.

Por último, creemos que el criterio de la Subtel atenta contra del espíritu de la Ley 20.453, al restringir la observancia de la neutralidad negativa, lo que deja a las normas que consagran la neutralidad positiva, tanto en la ley como en el reglamento, en calidad de letra muerta.

Lo anterior también explica por qué la Subtel no ha ejercido - a la fecha de este artículo- ningún procedimiento sancionatorio en contra de las empresas de telefonía móvil por la aplicación del zero-rating, según los datos entregados por el propio organismo a través de la ley de transparencia, y a pesar de los oficios enviados a todas las compañías telefónicas desde 2014. Esto, además, se extiende a la observancia en general de las obligaciones de fondo impuestas por el artículo 24.h de la ley, ya que de acuerdo con el análisis de Castro, Pereira da Silva y Viollier (2017: 31), de los 48 cargos presentados por el regulador contra las empresas de telefonía relativos a la neutralidad de la red, solo dos se refieren a obligaciones sustantivas - contenidas en sus literales a), b) y c)—, mientras que el resto sancionó las trasgresiones de los ISP a los deberes de información establecidos en la letra d del artículo 24.h.

En síntesis, el regulador ha seguido principalmente dos criterios respecto al zero-rating:

- Primer criterio (2010-2014): Prohibición de las prácticas de discriminación arbitrarias, aunque permite excepcionalmente a aquéllas que dieran el mismo trato - como, por ejemplo, la gratuidad - a todos los servicios de una misma naturaleza. Este criterio, contenido tanto en el reglamento como el Oficio 40, es coherente con el texto y el espíritu de la legislación.

- Segundo criterio (2015-2017): Aceptación del zero-rating en todos los casos, con única limitante de la prohibición de las acciones que impidieran el acceso (bloqueo) a los servicios de la igual naturaleza. Además de ello, la Subtel explicitó que la discriminación positiva quedaba fuera de su competencia, al ser materia de libre competencia, lo cual se ha visto reflejado en la nula actividad administrativa en relación con la tasa cero desde la vigencia de este criterio. 


\section{Conclusiones}

La neutralidad de la red es un concepto que incluye dos concepciones: la prohibición del bloqueo e interferencia del tráfico de internet (neutralidad negativa) y la discriminación y priorización de ciertos servicios respecto de otros del mismo tipo (neutralidad positiva). Si bien el zero-rating, al ser una práctica que implica discriminación tarifaria en el cobro por el uso de ciertos sitios o aplicaciones, podría configurarse como una vulneración per se a la neutralidad, la diversidad de modelos de tasa cero hacen necesaria una aproximación intermedia para su regulación, toda vez que hay prácticas que son denominadas como zero-rating pero que no atentan contra la neutralidad de la red.

A partir de nuestro análisis, podemos caracterizar a la legislación chilena de neutralidad de la red como una norma que recoge ambas concepciones (negativa y positiva) de dicho principio.

Si bien la norma, de manera aislada, permitiría colegir que en Chile se prohíbe de manera absoluta al zero-rating, lo cierto es que el regulador incorporó en el reglamento de la ley una definición de lo que constituye una práctica arbitraria, que implícitamente permite aquellas actuaciones de los ISP, entre ellas la tasa cero, que extiendan el mismo trato a todos los servicios del mismo tipo. El mismo criterio fue recogido por el regulador chileno en el oficio sobre zero-rating de 2014.

Sin embargo, desde 2015 la Subsecretaría de Telecomunicaciones ha instaurado un nuevo criterio, en que se prohíbe la tasa cero solo en los casos en que se bloqueen los servicios del mismo tipo, lo que en la práctica constituye la aceptación del zero-rating en todos sus modelos, lo cual solo ha profundizado la proliferación de esta oferta comercial en el mercado chileno.

El criterio cambiante del regulador podría suponer una crítica al modelo de cumplimiento de la legislación de neutralidad de la red en Chile, que recae en la Subsecretaría de Telecomunicaciones; sin embargo, dado que los otros dos modos de observancia (derecho de la competencia y organismos supranacionales) no son viables en el actual contexto chileno, creemos que el uso de la potestad sancionadora por el organismo administrativo sigue siendo la solución más adecuada.

La problemática en la aplicación de la ley de neutralidad radica en que la definición de las conductas prohibidas recae en el propio regulador, a través de su potestad reglamentaria, y no en la ley, que define ampliamente las conductas discriminatorias. Ello, en la práctica, permite e incluso fomenta que haya cambios en los criterios sobre el cumplimiento de la ley, como se ha analizado en este artículo con respecto del caso del zero-rating. Este inconveniente podría incluso profundizarse con cambios de funcionarios o de Gobierno, cuyo caso más representativo es el rechazo de la neutralidad por la FCC estadounidense durante el primer año del Gobierno de Donald Trump, lo que desechó una década de avances en la materia. 
El problema de las discrepancias entre la legislación y su ejecución pueden ser enfrentados mediante dos formas, que no son excluyentes.

La primera, de lege lata, recae en el organismo regulador, que debe establecer una política clara sobre el zero-rating, que sea congruente con la ley y su espíritu. Esto no implica necesariamente un cambio en el reglamento de la ley, sino que puede determinarse mediante un documento que aporte una carta de navegación a mediano plazo sobre la protección de la neutralidad de la red en Chile. Dicha política debería aportar ejemplos sobre cuáles modelos de zero-rating son atentatorios contra la neutralidad y cuáles no, de manera de orientar a los ISP y, a la vez, informar a los consumidores.

La segunda solución, de lege ferenda, es la proposición por el Ejecutivo o por los miembros del Congreso Nacional de reformas a la ley de neutralidad de la red que tengan como objetivo superar la ambigüedad de los verbos rectores de la normativa vigente, al establecer de manera expresa los conceptos de neutralidad positiva y negativa, en una forma análoga a la que se hizo en el reglamento..$^{8}$ De acuerdo con lo planteado en este artículo, creemos que una aproximación relativa de la legislación al zero-rating es la mejor fórmula para su regulación, sin prohibirla en todos sus casos.

Ambas propuestas, que no son mutuamente excluyentes, tienen como objeto que se otorgue certeza jurídica a los tres sujetos involucrados en la neutralidad de la red: a los ISP, para que puedan ofrecer prácticas comerciales atractivas dentro de un marco legal claro; a los proveedores de contenidos, para que se privilegien condiciones de igualdad y competitividad en el ambiente de internet que estimulen a la innovación en aplicaciones y sitios; y por último, pero no menos importante, a los usuarios, para que tengan la garantía de elegir en forma libre a cuáles contenidos acceden, sin que haya estímulos perversos de los otros dos sujetos para escoger sus servicios.

\section{Anexo 1: Oficios enviados por Subtel sobre zero-rating (2014-2017)}

La tabla 2 detalla los actos administrativos dictados por la Subsecretaría de Telecomunicaciones de Chile relacionados con el uso e implementación de la ley de neutralidad de la red, específicamente en lo relativo a la práctica del zero-rating.

38. Un proyecto de ley actualmente tramitado en el Congreso Nacional (Boletín 10.999-15) busca, entre otras modificaciones, incorporar al artículo 24.h el literal e): «Solo podrán dar un trato diferenciado a un contenido para ofrecer mejores condiciones de acceso o de uso para el usuario, sin embargo, deberán ofrecer el mismo trato a todos los contenidos de similar o misma naturaleza cuando así les sea requerido por un proveedor de contenidos. El mejor trato ofrecido no podrá ser objeto de un acto o contrato a título oneroso entre proveedores de acceso y contenido». 
Tabla 2. Oficios dictados por la Subtel sobre zero-rating

\begin{tabular}{|c|c|c|c|}
\hline Oficio & Fecha & Destinatarios & Objetivo \\
\hline Circular 40 & 14 de abril de 2014 & Varios & Imparte instrucciones al marco normativo vigente \\
\hline Ordinario 3.618 & 2 de mayo de 2014 & Virgin Mobile & Remite copia del 0ficio Circular 40 de 2014 \\
\hline Ordinario 5.074 & 4 de junio de 2014 & Claro Chile & Reitera instrucciones al marco normativo vigente \\
\hline Ordinario 5.075 & 4 de junio de 2014 & VTR Wireless & Reitera instrucciones al marco normativo vigente \\
\hline Ordinario 5.084 & 4 de junio de 2014 & Telefónica Móviles Chile & Reitera instrucciones al marco normativo vigente \\
\hline Ordinario 5.374 & 11 de junio de 2014 & Virgin Mobile & Reitera instrucciones al marco normativo vigente \\
\hline Ordinario 7.509 & 18 de agosto de 2014 & Claro Chile & $\begin{array}{l}\text { Instruye sobre la comercialización de promociones } \\
\text { y ofertas con acceso a redes sociales sin costo }\end{array}$ \\
\hline Ordinario 7.510 & 18 de agosto de 2014 & Telefónica Móviles Chile & $\begin{array}{l}\text { Instruye sobre la comercialización de promociones } \\
\text { y ofertas con acceso a redes sociales sin costo }\end{array}$ \\
\hline Ordinario 7.821 & 26 de agosto de 2014 & Netline Telefónica Móvil & Responde solicitud de pronunciamiento \\
\hline Ordinario 7.948 & 28 de agosto de 2014 & Virgin Mobile & $\begin{array}{l}\text { Instruye sobre la comercialización de promociones } \\
\text { y ofertas con acceso a redes sociales sin costo }\end{array}$ \\
\hline Ordinario 14.725 & 12 de noviembre de 2015 & Entel PCS & $\begin{array}{l}\text { Instruye sobre la comercialización de promociones } \\
\text { y ofertas con acceso a redes sociales sin descontar saldo }\end{array}$ \\
\hline Ordinario 7.502 & 16 de agosto de 2016 & WOM & $\begin{array}{l}\text { Instruye sobre la comercialización de promociones } \\
\text { y ofertas con acceso a redes sociales sin descontar saldo }\end{array}$ \\
\hline Ordinario 7.503 & 16 de agosto de 2016 & Entel PCS & $\begin{array}{l}\text { Instruye sobre la comercialización de promociones } \\
\text { y ofertas con acceso a redes sociales sin descontar saldo }\end{array}$ \\
\hline Ordinario 915 & 18 de agosto de 2016 & Entel PCS & $\begin{array}{l}\text { Solicita información y ordena adecuación respecto } \\
\text { de la comercialización de recargas ilimitadas }\end{array}$ \\
\hline Ordinario 9.585 & 11 de agosto de 2017 & Claro Chile & $\begin{array}{l}\text { Solicita información y ordena adecuación respecto } \\
\text { de la comercialización de planes ilimitados }\end{array}$ \\
\hline Ordinario 9.515 & 10 de agosto de 2017 & Entel PCS & $\begin{array}{l}\text { Solicita información y ordena adecuación respecto } \\
\text { de la comercialización de planes ilimitados }\end{array}$ \\
\hline Ordinario 11.182 & 20 de agosto de 2017 & Telefónica Móviles Chile & $\begin{array}{l}\text { Solicita información y ordena adecuación respecto } \\
\text { de la comercialización de planes ilimitados }\end{array}$ \\
\hline Ordinario 12.939 & 30 de octubre de 2017 & WOM & $\begin{array}{l}\text { Solicita información y ordena adecuación respecto } \\
\text { de la comercialización de planes ilimitados }\end{array}$ \\
\hline Ordinario 15.475 & 29 de diciembre de 2017 & WOM & $\begin{array}{l}\text { Solicita información y ordena adecuación respecto } \\
\text { de la comercialización de bolsas ilimitadas }\end{array}$ \\
\hline
\end{tabular}

Fuente: Elaboración propia. Información solicitada mediante Ley 20.285, Sobre Acceso a la Información Pública.

\section{Referencias}

ARD, BJ (2016). «Beyond neutrality: How zero rating can (sometimes) advance user choice, innovation, and democratic participation». Rayland Law Review, 75: 9841.028. Disponible en http://bit.ly/2trjuTE.

BARATA, Joan (2012). «El concepto de net neutrality y la tensión entre regulación pública y autorregulación privada de las redes». Internet, Derecho y Política, 13: 44-52. DOI: 10.7238/idp.voi13.1480. 
Brouwer, Dennis (2017). «Zero-rating and net neutrality in the European Union». Tesis de grado, Universidad de Tilburg, Alemania. Disponible en http://bit. ly/2IfSSd2.

CARrillo, Arturo (2016). «Having your cake and eating it too? Zero-rating, net neutrality and International Law». Stanford Technology Law Review, 19 (3): 364-429. Disponible en https://stanford.io/2IjdwJd.

Castro, Oona, Sivaldo Pereira da Silva y Pablo Viollier (2017). Neutralidad de red en América Latina: Reglamentación, aplicación de la ley y perspectivas. San Pablo, Santiago: Derechos Digitales. Disponible en https://bit.ly/2z3GbOO.

CoMisión EUROPEA (2017). Zero-rating practices in broadband markets. DOI: $10.2763 / 002126$.

EISENACH, Jeffrey (2015). The economics of zero rating. Nueva York: NERA. Disponible en https://bit.ly/29yVW6W.

Gharakheili, Hassan Habibi, Arun Vishwanath y Vijay Sivaraman (2016). «Perspectives on net neutrality and internet fast-lanes». Computer Communication Review, 46 (1): 64-69. DOI: 10.1145/2875951.2875962.

Huichalaf, Pedro (2015a). La neutralidad de la red: El caso chileno. Santiago: Subsecretaría de Telecomunicaciones. Disponible en https://bit.ly/2vz5OcR.

-. (2015b). «Neutralidad de la red: Explorando el impacto en REGULATEL». Disponible en https://bit.ly/2K3oncx.

JoRDAN, Scott y Arijit GHOSH (2010). «A framework for classification of traffic management practices as reasonable». Transactions on Internet Technology, 10 (3): 1-23. DOI: $10.1145 / 1852096.1852100$.

KeEnAN-AlsPeCtor, Jenna (2016). «What is free about free basics?». The 44th Research Conference on Communication, Information and Internet Policy 2016. DOI: 10.2139/ssrn.2756691.

Layton, Roslyn y Silvia Elaluf-Calderwood (2015). «Zero rating: Do hard rules protect or harm consumers and competition? Evidence from Chile, Netherlands and Slovenia». DOI: 10.2139/ssrn.2587542.

MARSDEN, Christopher (2010). Net neutrality: Towards a co-regulatory solution. Londres: Bloomsbury Academic.

-. (2016). "Comparative case studies in implementing net neutrality: A critical analysis of zero rating». Scripted, 13 (1): 1-39. DOI: 10.2966/scrip.130116.1.

Mors, Martin (2014). "Neutralidad de la red en Estados Unidos: De vuelta al pizarrón». Revista Chilena de Derecho y Tecnología, 3 (1): 139-161. DOI: 10.5354/0719-2584.2014.32223.

OCDE, Organización para la Cooperación y el Desarrollo Económicos (2016). Perspectivas de la OCDE sobre la economía digital 2015. Ciudad de México: Microsoft México. DOI: 10.1787/9789264259256-es. 
Rossini, Carolina y Taylor Moore (2015). Exploring zero-rating challenges: Views from five countries. Washington DC: Public Knowledge. Disponible en https://bit. ly/2G3vsLy.

Soares Ramos, Pedro (2014). «Towards a developmental framework for net neutrality: The rise of sponsored data plans in developing countries». 2014 TPRC Conference Paper. DOI: 10.2139/ssrn.2418307.

VAN SCHEwICK, Barbara (2010). «Network neutrality: What a non-discrimination rule should look like». Stanford Public Law Working (inédito). DOI: 10.2139/ ssrn.1684677.

Wu, Tim (2003). «Network neutrality, broadband discrimination». Journal of Telecommunications and High Technology Law, 2: 141-176. DOI: 10.2139/ssrn.388863.

\section{Agradecimientos}

Este trabajo presenta los resultados del proyecto «Zero rating and net neutrality: The state of the art in Chile», financiado por la Internet Policy Observatory de la Annenberg School for Communication (Universidad de Pensilvania, Estados Unidos). El autor agradece la mentoría de Jan Gerlach (PhD, Universidad de San Galo, Suiza).

\section{Sobre el autor}

Marco Correa Pérez es abogado. Licenciado en Ciencias Jurídicas y Sociales de la Universidad de Chile. Profesor del Diplomado de Comunicación Digital del Instituto de Comunicación e Imagen de la Universidad de Chile. Presidente de la Corporación Wikimedia Chile. Su correo electrónico es legal.mcorrea@gmail.com. 


\title{
REVISTA CHILENA DE DERECHO Y TECNOLOGÍA
}

La Revista de Chilena de Derecho y Tecnología es una publicación académica semestral del Centro de Estudios en Derecho Informático de la Facultad de Derecho de la Universidad de Chile, que tiene por objeto difundir en la comunidad jurídica los elementos necesarios para analizar y comprender los alcances y efectos que el desarrollo tecnológico y cultural han producido en la sociedad, especialmente su impacto en la ciencia jurídica.

\author{
EDITOR GENERAL \\ Daniel Álvarez Valenzuela \\ (dalvarez@derecho.uchile.cl) \\ SITIO WEB \\ rchdt.uchile.cl \\ CORREO ELECTRÓNICO \\ rchdt@derecho.uchile.cl \\ LICENCIA DE ESTE ARTÍ́CULO \\ Creative Commons Atribución Compartir Igual 4.0 Internacional
}

\begin{abstract}
y
La edición de textos, el diseño editorial

y la conversión a formatos electrónicos de este artículo

estuvieron a cargo de Tipográfica

(www.tipografica.cl).
\end{abstract}

